\title{
Orientation-Based Interaction on Mobile Devices and Desktops - An Evaluation
}

\author{
Johannes Schwank, Franca-Alexandra Rupprecht, and Sebastian Schöffel \\ Computer Graphics and $\mathrm{HCl}$, University of Kaiserslautern \\ \{schwank, rupprecht, schoeffel\}@cs.uni-kl.de
}

Keywords: Interaction; Input metaphor; Mobile Devices; Tablets; Orientation-based; Hand gesture recognition; Leap Motion; Evaluation.

\begin{abstract}
Nowadays, mobile devices, such as smartphones, tablets or smartwatches, are essential items in our daily life. Further, more and more people use smart mobile devices in their everyday work for remote controlling, observing diagrams, performing web analytics, etc. However, the full potential of mobile devices is not tapped yet; built-in sensors such as accelerometers or gyroscopes offer a wide range of interaction capabilities, which are still often not fully used in nowadays mobile applications. On the other hand, desktops are still the dominating working device, but with significantly differing interaction means. With additional hand tracking devices capturing the user's gestures additional input possibilities are available but still often unused.

In this paper, we investigate on a concept for orientation-based touch-less interaction. Depending on the type of device - traditional desktop or a mobile device - we use an interaction metaphor called "Waggle"; utilizing of tilting and turning of either the user's hand or the mobile device itself for additional input possibilities. Based on the results of two pilot studies for both environments, basic parameters for future design decisions are derived: on the one hand, the maximum angle for basic rotation axes are evaluated. On the other hand, different discretizations of tilt and turn angles are investigated. Based on the outcome of both studies the optimal configuration for the use of the Waggle interaction metaphor in future applications on both mobile and desktop environments are defined.
\end{abstract}

\section{Introduction}

Industrial corporations comprise many different disciplines from mechanical engineering to industrial engineering up to computer science. Additionally, industrial corporations can be adapted to a layer model, where every layer is considered as one subsystem that is in an exchange relationship with the neighboring levels. Design decisions on the process level (the lowest level) escalate via the machine level up to the factory level (the highest level) and vice versa. It is easy to see that such a system is a synergy and functional interaction of many different aspects and interconnections.

It is nearly impossible to represent all these aspects in one view that is still able to provide all necessary details and also does not overwhelm the user with unnecessary information. Conclusive,

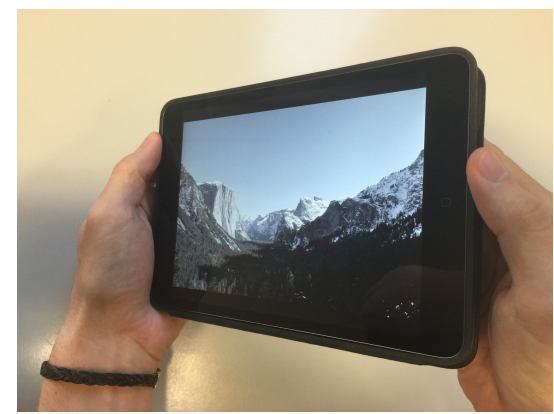

(a)

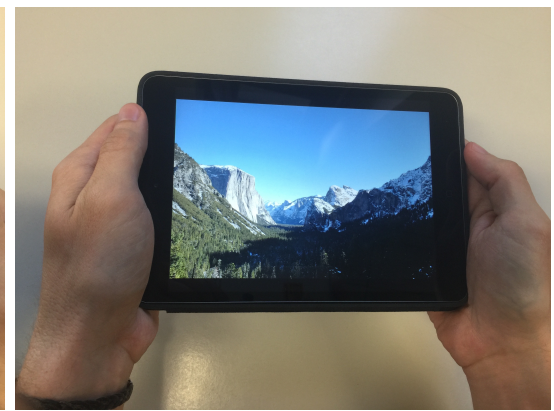

(b)

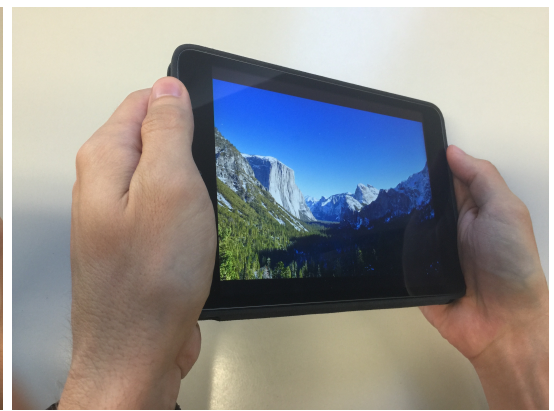

(c)

Fig. 1: The waggle interaction allows users of mobile devices to select values or to change viewports on the visualized data. 
it is highly challenging to observe several facets of the system with the aim to get an all-embracing impression on the ongoing processes. Implying the presence of different views on the system, intuitive visualization and interaction techniques are needed.

Established interaction approaches on smart devices feature touch gestures in combination with elements such as buttons or sliders. Adding additional UI-elements to the very limited display space leads to an even more shrinked viewport and hidden information. Furthermore, while performing touch gestures, the fingers may also hide important information. A touchless interaction overcomes these issues, but it is to examine if such an interaction is applicable on mobile smart devices.

The principle of a multiscopic image (visualization of two or more images, each visible due to the user's head position) can be adapted to mobile devices using built-in sensors such as accelerometer and gyroscope. This enables tilting as a touchless orientation-based interaction for displaying different views or adjusting parameters.

In desktop environments, it is obviously not possible to change the orientation of the monitor to adjust the view and thereby applying the multiscopic image principle. However, a comparable interaction metaphor can be achieved by replacing the mobile device such as tablets by the user's hand. Appropriate devices that support the recognition of hand orientation are already very precise and reasonably priced.

In this paper, we present the so-called Waggle metaphor - an orientation-based method to interact with mobile smart devices by enabling multiple views on the underlying data by changing the inclination of the device [14]. We extend this approach for the use in desktop environments by replacing the device orientation by the hand orientation. Our contribution are two pilot studies that provide data of the maximum tilt angle and step interval size to gain the optimal configuration for the Waggle interaction on tablets and in desktop environments. Finally, we compare the results and the resulting configurations regarding mobile and desktop environment.

\section{Related Work}

Considering mobile devices and the angle of the viewpoint to the display, lenticular lenses are one of the first thoughts. In general, they are used to generate glasses-free, auto-multiscopic 3D images. Special lenses are set in a way that two stripes (one of each images) are covered. Depending on the viewing angle, only one image can be seen [23]. One of the disadvantages is that the two images have to be already calculated in advance. In our approach, with each change of the viewing angle, the displayed image is adjusted. Translating to the desktop environment, it is the user's hand orientation instead of the viewing angle which is changing.

Tilting and turning as interaction metaphor of mobile devices finds relevance in the work of $[2,15$, $8,12,10]$. Baglioni et al. [2] analyzed device tilting interaction using accelerometer. They found out that the tilt gestures are additional input channels that do not interfere with other input gestures like touch. While they give an overview over accelerator based input techniques like shaking the device (e.g. skip the next song on Apple's iPod), many examples where tilting is used as additional input metaphor are collected by Scoditti et al. in [15]. Rekimoto investigates tilting interaction to select items from linear and circular menus [11]. In his approach, tilt angles are associated to menu items. In [8], Partridge et al. describe TiltType, a technique that supports text input. They correlate tilt angles to letters that are organized in circular menus. Roudaut et al. [12] proposed to use tilting to switch between applications. Tapping the device's back side activates the switching mode while tapping on the front side the desired application is selected.

Rahman et al. [10] defined the range-of-motion i.e. the rotation of the wrist along each axis. They motivated also the need to specify a discretization of the tilt angles, which can directly influence the tilt interaction. In their evaluation, linear discretization is compared to sigmoid and quadratic discretization. They found out that "users can control comfortably at least 16 levels on the pronation/supination axis and that using a quadratic mapping function for discretization of tilt space significantly improves 


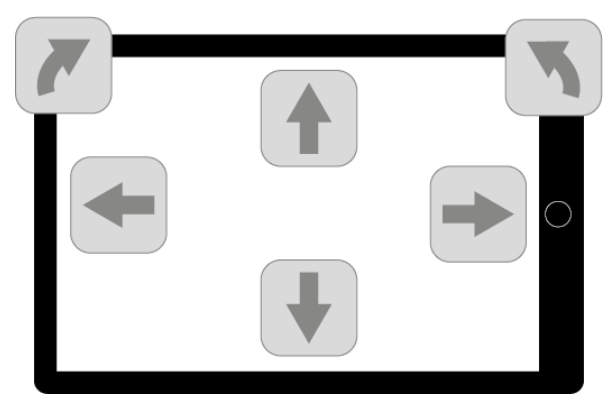

Fig. 2: 6 simple filters based on 3 DOF on smart device.

user performance across all tilt axes.". Most of the work such as $[2,10,12]$ targets one-handed interaction. But since tablets becoming more popular nowadays and are mostly held with two hands, the two-handed interaction is getting interesting and has not been investigated in detail. Therefore, we extend the above mentioned work and examine the tilting and turning technique further.

In desktop environments, a technique to observe the user's hand's orientation is required. This can be done by using the Leap Motion controller [6]. The Leap Motion controller is an infrared-based sensor developed by the Leap Motion, Inc. The main purpose of this device is to track hand and finger position and orientation relative to the device centered coordinate system. Besides three basic gestures such as swipe, tap, and rotate, the provided API allows access to a lot of extracted features (e.g. left hand/right hand, palm position, individual finger direction, confidence, etc.), however the raw infrared image is not accessible.

Physical limits of hand rotation are given in different medical contexts [3, 13]. The high precision and reliability of the device could be verified [4, 21, 9]. Combining the Leap Motion controller with the Microsoft Kinect Sensor can improve the recognition [7]. Bachmann et al. [1] and Seixas et al. $[17,16]$ investigated the usability of the Leap Motion controller for pointing tasks and compared it to mouse or touch pad interaction. Both of them conclude that in terms of effectiveness and efficiency the touch-free Leap Motion interaction will neither replace the mouse nor the touch pad. Jakus et al. report an insufficient accuracy in border regions of the motion space and varying sampling rates which leads them to the conclusion that the Leap Motion controller should not yet replace professional hand tracking systems [5]. A possible use case of incorporating the user's hand in applications is mentioned in [19], Shen et al. propose to use the hand orientation as indicator for clipping plane in 3D medical images.

\section{Concept}

The initial viewpoint shows the data-set without any filters and layers. Depending on the environment, tilting the hand (in desktop environment) or the device (in mobile environment) along one of the axes (longitudinal, lateral, or perpendicular axis) filters the respective overlapped layers. This results in 3 degrees of freedom (DOF) providing 6 simple filters as shown in Fig. 2 and Fig. 3. Furthermore, the observed object (hand or device) can be tilted along up to all three axes at the same time, e.g. along the longitudinal axis and turning around the perpendicular axis. Overall, this results in a possible definition of 6 simple filters and 20 filter combinations, i.e. in total 26 different views (see Fig. 4).

The approach of an orientation-based interaction metaphor for modern mobile devices as described in [14] uses the built-in accelerometer and gyroscope. The viewing angle is solely changed by using the data generated by the built-in accelerometer and the gyroscope of the used device. The translation of the waggle metaphor to the desktop environment requires other sensors. Instead of taking the device orientation, because it is not possible to tilt the monitor in a comparable way like a tablet, we identify the user's hand orientation using the Leap Motion controller [6]. Thereby, the concept of [14] can basically reused. . 


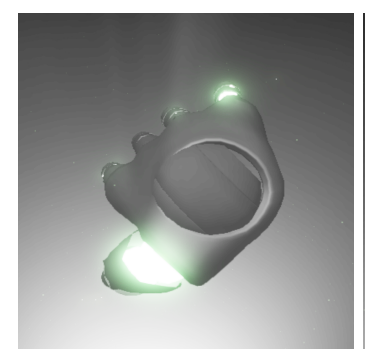

(a)

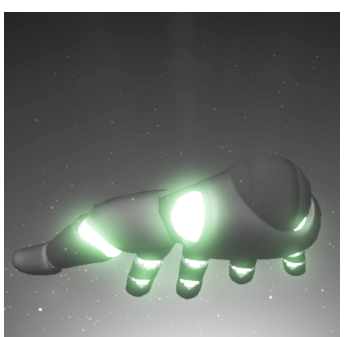

(d)

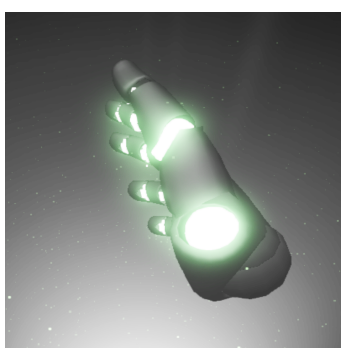

(b)

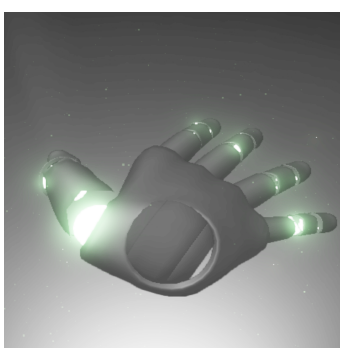

(e)

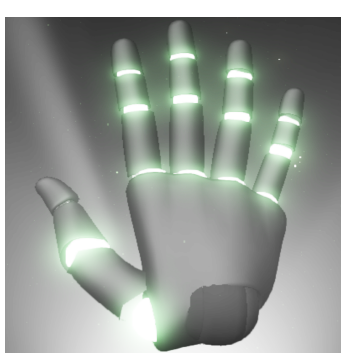

(c)

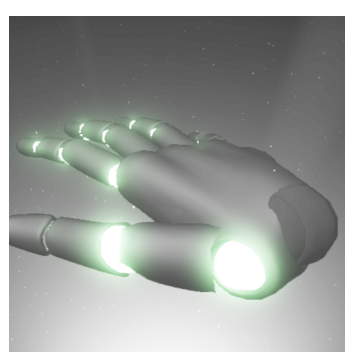

(f)

Fig. 3: 6 simple filters based on 3 DOF of the hand, measured by the Leap Motion controller. (a) roll left (b) roll right (c) pitch up (d) pitch down (e) yaw right (f) yaw left

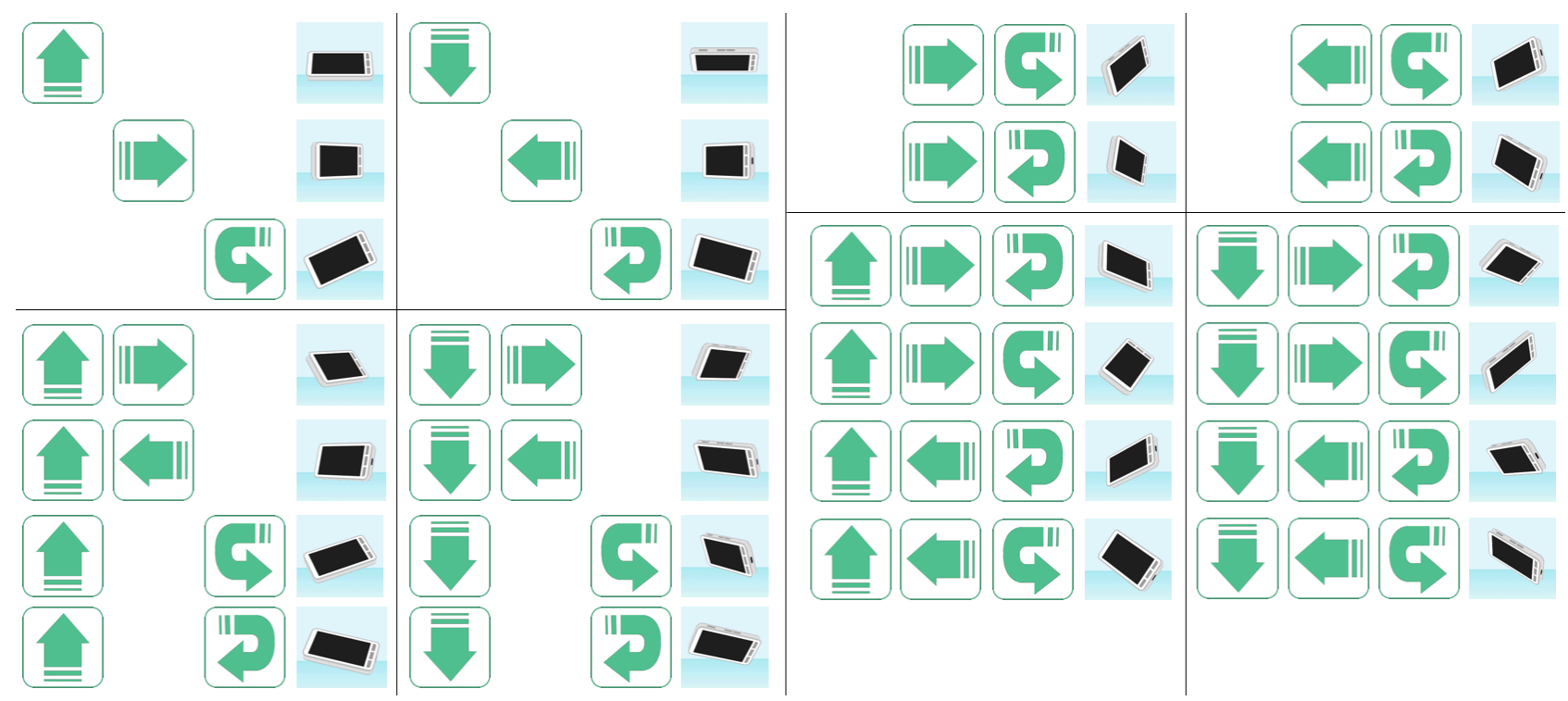

Fig. 4: Waggle directions. This provides 6 simple filters (rows 1-3). Combinations of them enable additional 20 possibilities. Example: tilt the device to the left and turn it clockwise. Notice, the mobile device can be replaced by the user's hand. 
A lock mechanism as described in [14] is useful in a desktop environment as well. However, handing over the full desktop station is not possible, but still, by locking the current orientation, the user is able to use both of his hands again to control mouse and keyboard (or other input devices). The lock mechanism can be activated via keyboard command.

The applied range of motion and discretization are the key-players for providing a high usability of our concept. Consequently, we examined those values in more detail in two pilot studies in both environments and discuss on the results.

Range of motion. The range of motion is defined by the maximum tilt and turn angle in each direction in a way that a user is still able either to handle the device or move his hand comfortably. As described in [14] in detail, the first pilot study evaluated the range of motion for two-handed device control. We examined if the maximum tilt and turn angles correspond to the range of motion of the one-handed device control that was found by Rahman et al. [10]. The pilot study also investigated if the maximum angles are depending on the tilt and turn direction. In the paper at hand, we reperformed the study using the Leap Motion controller in desktop environment and compared the results of the two different scenarios.

Discretization. The subdivision of the range of motion is an important usability factor. Current mobile devices feature accelerometers and gyroscopes that are able to measure the tilt angles with high precision. In fact, these measurements are so precise that the tilt angles change even though the user thinks that the device is in a stable position. The same holds for the use of the Leap Motion controller. Even if the user thinks that his hand is resting in the same position, the controller observes small changes. Hence, for our purpose, the values of the range of motion need to be discretized.

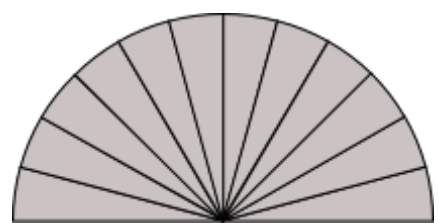

(a)

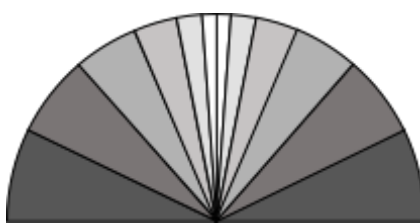

(b)

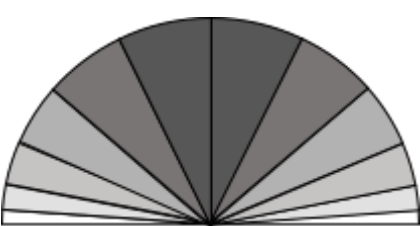

(c)

Fig. 5: Linear, quadratic and sigmoid discretization of a $180^{\circ}$ angle range as adapted from [10].

\section{Experimental User Studies}

The waggle interaction technique needs a good and intuitive definition of the maximum angle in each direction, the minimal interval size of the steps and the discretization of the range of motion. Therefore, we conducted two pilot studies. The first pilot study evaluated the maximum angle of each tilt and turn direction. In the second pilot study, we investigated the minimal interval size and the optimal discretization of the range of motion.

\section{Pilot Study 1}

The goal of the first pilot study was to get insight in the maximum angle reachable by a human user. The participants of the study had to tilt or turn the device resp. their hand five times in each direction. For each task, we stored the start and maximum angle values of the relevant waggle direction.

Waggle on Mobile. This study answers the question how far can the user tilt the device and is still able to look at and interact with the device in a comfortable way when holding it with both hands (Figure 6). As there are many different mobile devices nowadays with different sizes, we selected four devices to evaluate if differences related to their size exist: the iPhone 4 (3,5"), the iPhone $6+(4,7$ "), the iPad mini (7,9") and the iPad2 (9,7").

Each participant had to perform 30 tasks per device, thus, 120 tasks overall, where the order in which the participants used the devices was random. 


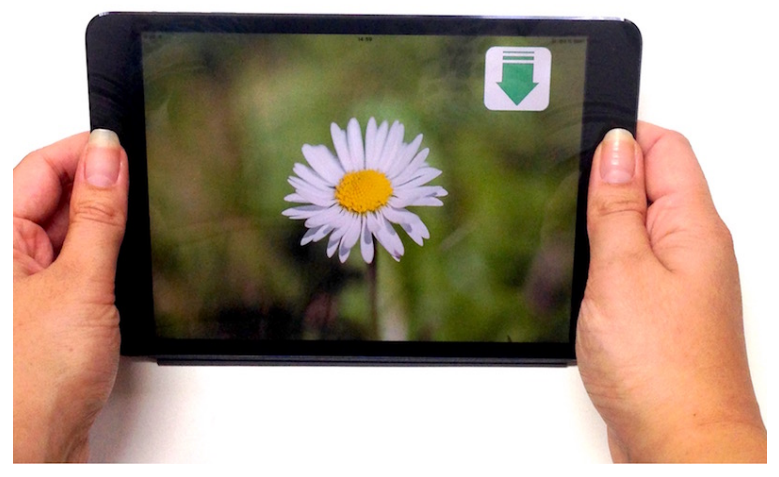

(a)

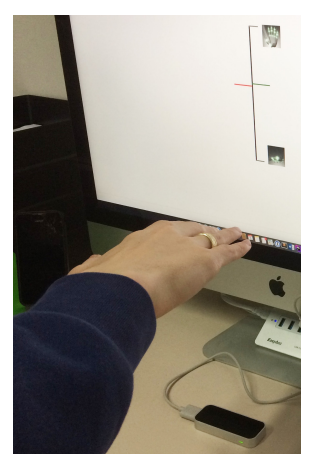

(b)

Fig. 6: Pictures of a participants in our pilot studies (a) using mobile devices and (b) using hand recognition with the Leap Motion in desktop environment.

The 15 participants of the first pilot study (4 female and 11 male regular smart device users) were asked to turn and tilt the device following the waggle direction that was displayed as an arrow on the device (longitudinal, lateral, or perpendicular axis). The participants were asked to stop when they do not feel comfortable anymore looking at the screen. To save that maximum angle, the participants performed a single tap anywhere on the screen. Then, they should return to their normal device position and tap again. We asked them to sit in their usual working position and not move their head, keep both hands on the device, and tap with one of their thumbs.

Results. Depending on the predefined Waggle direction, we calculated the difference between start and maximum angle of the appropriate coordinate, i.e. x-coordinate when pitching up and down (longitudinal axis), y-coordinate when rolling left and right (lateral axis), and z-coordinate when yawing the device clockwise and counterclockwise (perpendicular axis). We computed the median and the 25\%- and 75\%-quartiles (Q1 and Q3) for each direction and device. The analysis of these results (see Figure $7,8,9$ ) shows the median maximum angle for each direction. A detailed analysis of the angles using mobile devices can be found in [14].

Overall, to make the approach as applicable as possible, we set the maximum angles for both directions of the same axis to the same minimum value to not exclude left- or right-handed people and included a buffer where possible. The resulting ranges of motion can be seen in Table 1.

Waggle on Desktop. To find the maximum angle of hand rotation without a mobile device, we used the Leap Motion controller. This results in the research question how far can the user tilt his hand while it is still comfortable and does not force the user move more than his arm.

The pilot study 1 with the Leap Motion has been set up in a similar way as in the study with the tablet to enable comparison. Instead of two hands holding the device, this experiment was done with only the right hand. For our experiment, we defined a normal position, that means the palm points downwards. This has been the start position for the study. Figure 6(b) shows the setup of the study as well as the user's hand in normal position.

However, the task description was to pitch, roll or yaw until the individual maximum, but still comfortable position. The display showed the participant the direction how to turn or tilt his hand. As soon as he thought he reached the maximum angle, the participant was able to save the current angle of his hand's palm by pressing the space bar on the keyboard with the other hand. After each task, the hand should return to the normal position. This was always instructed via the display as well. Back in normal position, the participant started the next task by pressing the space bar again. Each participant had to tilt or turn his hand five times in each direction; the order was randomized. Thus, 


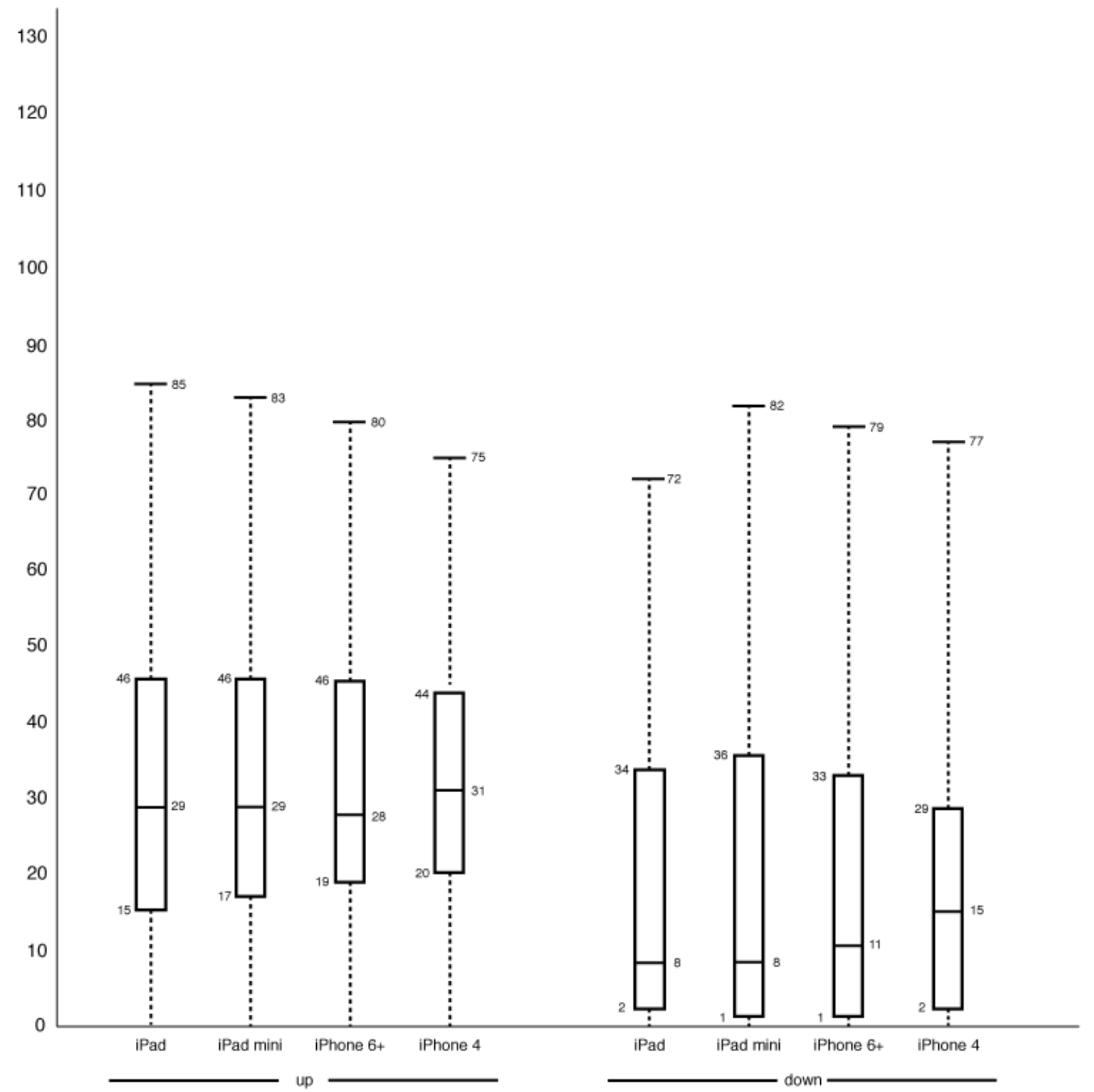

Fig. 7: Boxplot of the results of the first pilot study in mobile environment: lateral axis (pitching up and down). 


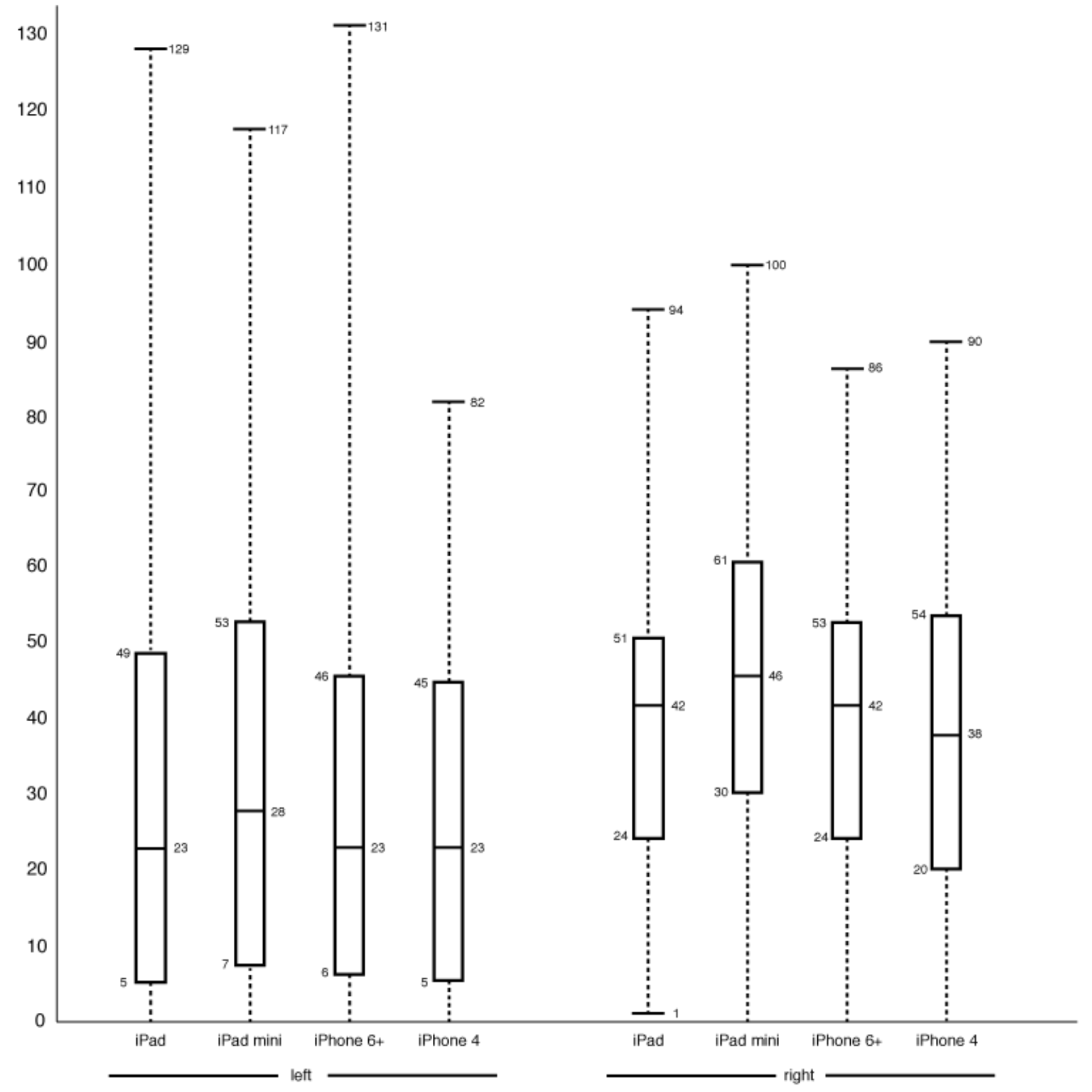

Fig. 8: Boxplot of the results of the first pilot study in mobile environment: longitudinal axis (rolling left and right). 


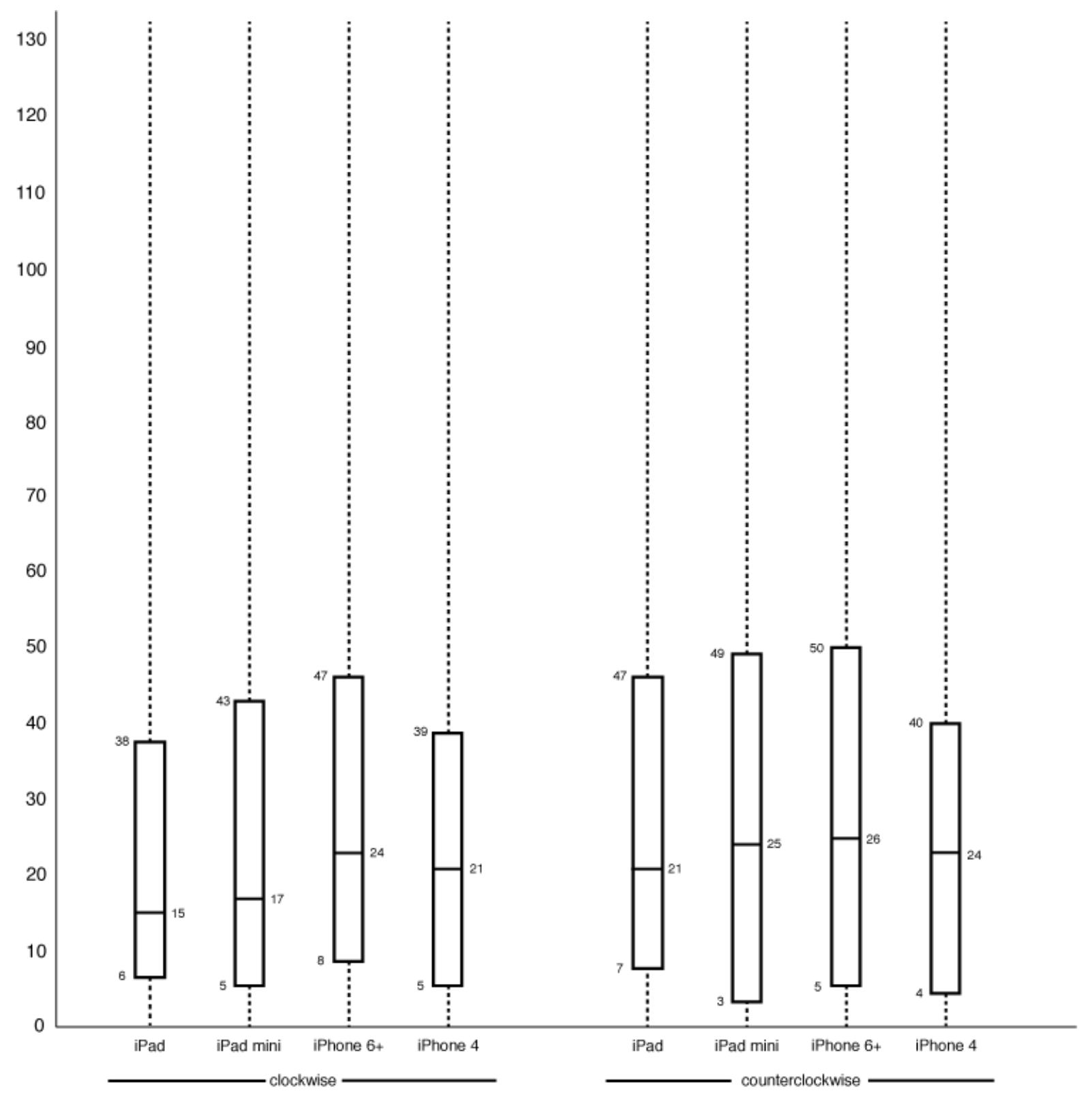

Fig. 9: Boxplot of the results of the first pilot study in mobile environment: perpendicular axis (yawing clockwise and counterclockwise). 
the total number of task per participant was 30 (according to the number of tasks using Waggle on mobile for one device).

Results. Overall, 13 males and 2 females participated in this pilot study, 12 of them were right-handed, 3 were left-handed. The box plot in Figure 10 shows the distribution of the stored values which where marked as maximum angle by the participants.

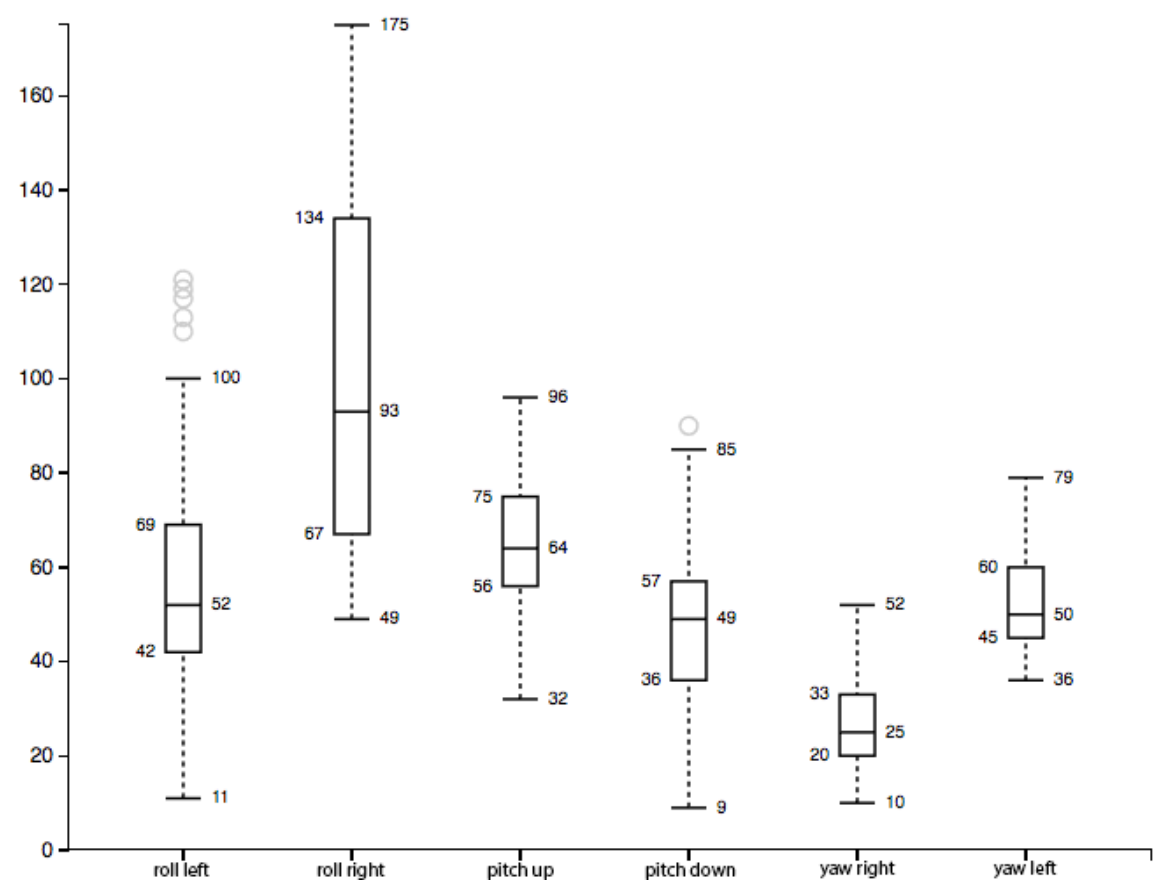

Fig. 10: Boxplot of the results of the first pilot study with the Leap Motion.

Considering the two left-most boxes (rolling the hand left and right around the longitudinal axis), it is noticeable that rolling right created higher angle values and the range is larger than the range of rolling left. The minimum value of the left roll angle after removing outliers is 11 , in contrast, the minimum value of the right roll angle after removing is 49. Compared to the physical limits as described in [3], our range of motion is significantly smaller. Their measuring was based on the neutral position of the hand (handshake position) leading to 90 degrees in each direction. Contrary, our measuring was based on normal position (hand palm face down), so that the hand is already rotated by 90 degrees. Considering that, it is obvious that it is not comfortable to roll the hand in this direction (roll left) furthermore. However, rolling right was expected to result in higher angles.

The results for pitching the hand up and down are shown in the third and fourth box of the box plot (see Figure 10). As described by Ryu et al. [13], the range of motion for daily activities for extension (pitch up) and flexion (pitch down) is 40 degrees. In our box plot, the minimum values of the maximum pitch angles are 32 (up) and 9 (down) degrees.

When yawing the hand clockwise and counterclockwise (ulnar and radial deviation), the physically maximum angle is at 30 (clockwise) and 20 (counterclockwise) degrees of turning [3]. In our experiment we got 10 and 36 degrees as minimums as shown in the box plot in the two right-most boxes. Surprisingly, the minimum in our experiment for the radial deviation is higher than the physically maximum. However, we could explain this by observing the participant's movements. As their arm were "free", the elbow could move to the side and provide more range of motion to the hand.

The overall concluded range of motion using the right hand can be seen in Table 1.

Discussion on Pilot Study 1. Comparing the results of pilot study 1 performed within both setups (Table 1), we can conclude that the resulting total range of motion in desktop environment is wider than in the mobile setup. Each rotation angle boast a wider range of motion within the usage of the 
Leap Motion controller, e.g. the total range of motion on the lateral axis in mobile environment is $32^{\circ}$ $\left(16^{\circ}+16^{\circ}\right)$, whereas in the desktop environment a range of $41^{\circ}\left(32^{\circ}+9^{\circ}\right)$ can be achieved.

Table 1: Range of Motion as the result of pilot study 1.

\begin{tabular}{l||r|r||r|r}
\multicolumn{1}{l||}{ axis } & \multicolumn{2}{c||}{ Mobile } & Desktop (right hand) \\
\hline \hline lateral axis (pitch) & up: $16^{\circ}$ & down: $16^{\circ}$ & up: $32^{\circ}$ & down: $9^{\circ}$ \\
\hline longitudinal axis (roll) & left: $20^{\circ}$ & right: $20^{\circ}$ & left: $11^{\circ}$ & right: $49^{\circ}$ \\
\hline perpendicular axis (yaw) & cw: $15^{\circ}$ & ccw: $15^{\circ}$ & cw: $10^{\circ}$ & ccw: $36^{\circ}$ \\
\hline
\end{tabular}

Consequently, if one wants to make use of these findings in an application, which should be applicable in mobile as well as in desktop environments with the same interaction metaphor, the ranges have to be matched. One possible solution is to cut the range of motion that is achieved within the Leap Motion controller setup. By doing so, a wide range of possible interaction capabilities is getting lost. It would be more reasonable to develop a mapping function between both achieved ranges of motion.

Without further evaluation, a wider range of motion leads to the assumption, that more steps in the whole interval are available. They can be used to enable a more granular control with the usage of the leap motion controller. However, since there is already a difference between the results of pilot study 1 depending on the environment, a second study is important to investigate the precision in mobile and desktop environments. In order to gather the actual step size and confirm respectively decline the assumption stated above, the following second study has been conducted.

\section{Pilot Study 2}

In this pilot study, we evaluated different discretizations of the range of motion based on the results of pilot study 1. Especially, we tried to answer how accurate a user can select a specific value by using the waggle metaphor with both a mobile device and the Leap Motion controller, that means what is the minimal interval size.

In pilot study 1, we could observe that the results regarding the single devices are similar enough to assume that the further investigation will not get falsified if we merely use one device type. We decided to use the iPad mini, which holds the average display size of all used devices. With the second pilot study, we wanted to investigate on what is the minimal size of one interval so that the user can still reach a specific angle by tilting or turning the device. Therefore, we used the results of the first pilot study to define the range of motion.

Based on the work and results of Rahman et al. [10], we applied linear discretization as well as a quadratic like and a sigmoid like discretization in our second pilot study. Overall, we evaluated five different discretizations (see Figure 5):

- linear1, linear2, linear4: The size of the intervals is 1,2 , and 4 degrees, respectively, over the whole range of motion.

- sigmoid: The interval size differs depending on the angle. The intervals around 0 are larger and becoming smaller the more they are away from 0 .

- quadratic: The interval size also depends on the angle, but is the opposite way of the sigmoid discretization, i.e. the greater is the distance to 0 , the greater the interval.

Thus, we get five task groups (linear1, linear2, linear4, sigmoid, and quadratic) for the study.

Waggle on Mobile. In each task group, the participants had to pitch, roll or yaw the device in each of the six simple directions five times resulting in 30 tasks per task group; overall 150 tasks per participant. The order of the tasks was randomized as well as the target values (within the range of motion).

The participants were asked to manipulate the device to match the current and the target value shown on the screen (see Figure 11). By holding the device two seconds in the desired position, we 
could assume that the position was stable and has been reached intentionally. We stopped the time for each task. After the experiment, the participants answered a questionnaire for gaining subjective feedback about the preferred interval size.

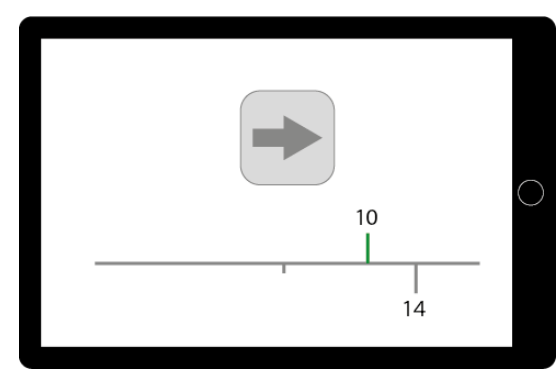

(a)

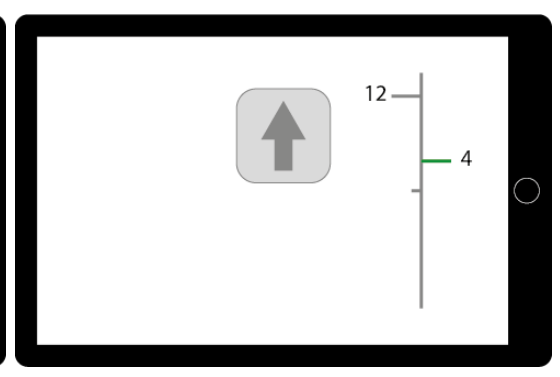

(b)

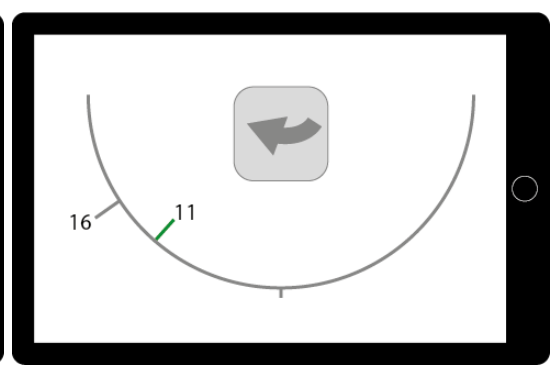

(c)

Fig. 11: UI-concept for the pilot study 2. Arrows show target direction, green marker represents the current angle. (a) Roll around longitudinal axis. (b) Pitch around lateral axis. (c) Yaw around perpendicular axis.

Results. In this second user study using Waggle on mobile, 14 participants ( 3 female, 11 male) took part. 10 of them were right-handed and 4 were left-handed. The overall average time to finish the experiment was 546,6 seconds, which leads to 3,64 seconds per task. In fact, the average times for the different directions do not differ significantly (Table 2) except for yawing around the $\mathrm{z}$-axis in linear1 tasks.

Table 2: Results of pilot study 2 using mobile device: Average time in seconds for each direction and task group.

\begin{tabular}{|c|c|c|c|c|c|}
\hline & $\begin{array}{l}\bar{\Xi} \\
\stackrel{\Xi}{\Xi}\end{array}$ & $\begin{array}{l}\stackrel{\Xi}{\Xi} \\
\stackrel{\Xi}{\Xi}\end{array}$ & $\begin{array}{l}\stackrel{+}{\Xi} \\
\stackrel{\Xi}{\Xi} \\
. \Xi\end{array}$ & 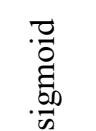 & 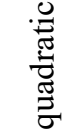 \\
\hline lateral axis (pitch) & $5,13 \mathrm{~s}$ & $2,44 \mathrm{~s}$ & $3,05 \mathrm{~s}$ & $3,05 \mathrm{~s}$ & $3,43 \mathrm{~s}$ \\
\hline longitudinal axis (roll) & $4,44 \mathrm{~s}$ & $2,02 \mathrm{~s}$ & $3,14 \mathrm{~s}$ & $3,64 \mathrm{~s}$ & $3,61 \mathrm{~s}$ \\
\hline perpendicular axis (yaw) & $7,10 \mathrm{~s}$ & $1,89 \mathrm{~s}$ & $4,25 \mathrm{~s}$ & $3,70 \mathrm{~s}$ & $3,75 \mathrm{~s}$ \\
\hline average & $5,56 \mathrm{~s}$ & $2,12 \mathrm{~s}$ & $3,48 \mathrm{~s}$ & $3,46 \mathrm{~s}$ & $3,60 \mathrm{~s}$ \\
\hline
\end{tabular}

As shown in Figure 12, the tasks were solved significantly faster with the linear2 discretization than with any other discretization. As observations have shown, the more the participant tilted the device, the more unstable was the control. In a short informal interview, one participant said, that "the jitter in the high value areas with the smallest step size is too strong to be able to reach a value precise, fast, and stable". Based on that, we expected that the linear4 discretization has to be even faster than the linear2 discretization. However, it was not the case. Our results, as described in [14], have shown that linear2 is better for two-handed tablet usage.

Waggle on Desktop. In general, we tried to build the same setup for evaluating the Waggle technique on desktops and therefore used a browser based evaluation framework.

The participants hold their right hand above the Leap Motion controller. By pitching, rolling or yawing their hand (depending on the shown task), they had to match the green bar to the red bar and hold it for two seconds. Figure 6(b) shows one example participant.

Five different task groups - one per discretization - with 30 tasks each had to be performed by each participant (with a random task group order and random target values). Again, we stopped the time for each task and calculated the mean value and the standard deviation during the two-second-period. By this, we were able to evaluate the stability of the hand. We set a timeout of 30 seconds for each 


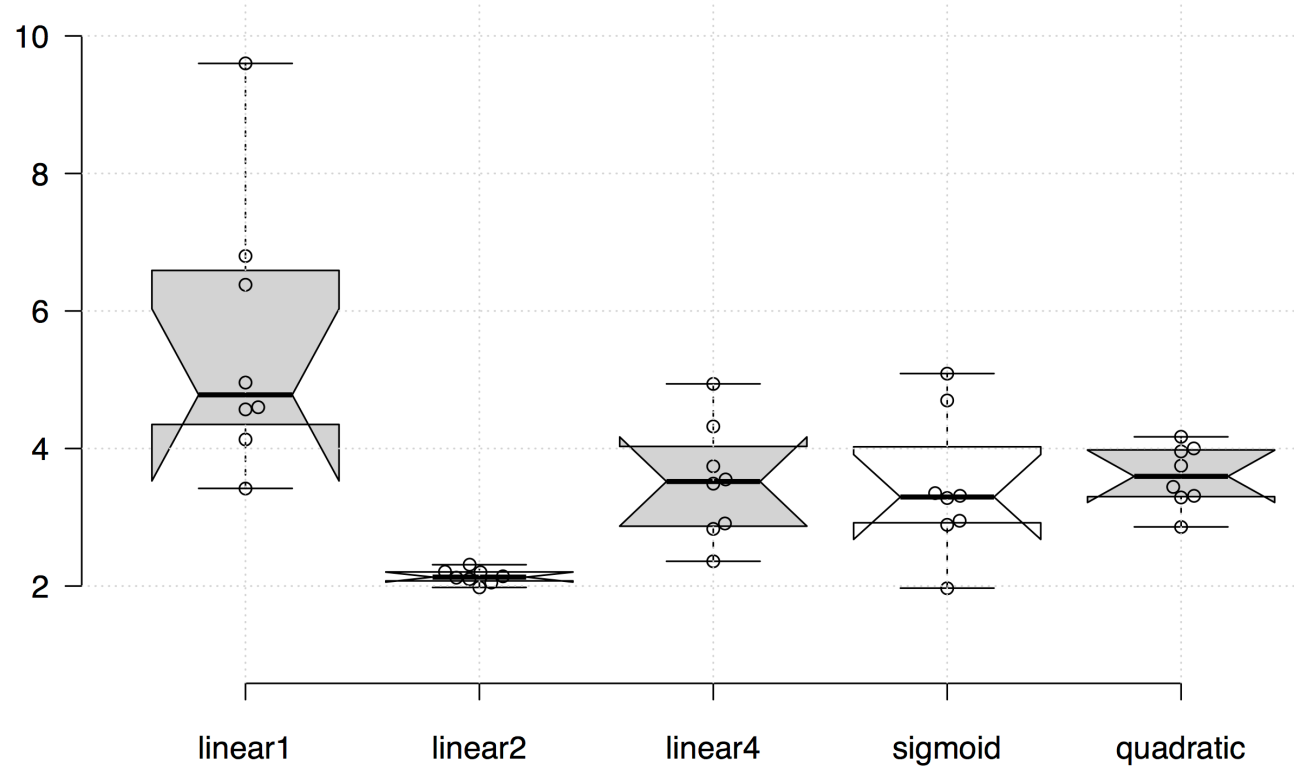

Fig. 12: Boxplot of the results of the second pilot study with mobile device.

task. If the task was not solved in this time period, it has been marked as not solved and the next task has been presented.

Results. The second pilot study using Waggle on desktop has been performed by 2 female and 11 male participants in the age between 21 and 29. Overall, the mean time to complete the study was 14 minutes and 56 seconds. The average times per task depending on its setting, the number of accepted and timeout tasks and the overall acceptance rate can be seen in Table 3. Altogether, 1950 tasks were performed and only 23 of them $(1,18 \%)$ were marked as timeout. In detailed analysis, we could observe that linear1 leads to the most timeouts.

The linear4 discretization has been the fastest in terms of average time needed for solving the tasks (4,06 seconds), whereas linear1 has been the slowest $(8,64$ seconds). The linear 2 discretization has been 1,15 seconds slower than linear4. Additionally, the acceptance rate is over 99\% (only 2 timeouts) which is still acceptable. This could be a trade-off solution between higher granularity of the range of motion and the speed of the selection. Beside the slow speed of linear1, there are the most timeouts using this discretization (13). Following these results, in our opinion, linear1 is not suitable.

Quadratic and sigmoid like discretization did not provide faster or more accurate results than the linear4 and linear2 discretization. Therefore, we do not see any advantage in contrast to Rahman et al. [10]. In the study, we asked all participants (right-handed and left-handed) to perform the tasks with their right hand. We could not find a significant difference in the measurements between left-handed and right-handed participants, as we could within the same study performed with the mobile device.

In fact, the Leap Motion sensor has such a high precision, so that there is an uncertainty due to the jittering of the user's hand. That might explain why linear1 and linear2 tasks were harder to solve for the participants, which means, the larger the interval size the faster the task solution, i.e. the selection of a value. A sigmoid or quadratic like discretization using a greater minimal interval step (e.g. as in linear4) could lead to better results (faster and less timeouts).

In the questionnaire following the 150 tasks, 3 participants mentioned that during the experiment over the time their arms and hands were getting tired and it was straining at the end. An integration of the waggle interaction in their daily work would be acceptable only for short interaction sequences.

Discussion on Pilot Study 2. As we observed in the study performed on the desktop environment, the task completion time decreased with the increasing of the linear discretization, meaning the discretization size of linear4 requires the shortest completion time. In contrast to that, in mobile environments, 
Table 3: Results of pilot study 2 using Leap Motion: Average time in seconds for each direction and task group. A task has been counted as accepted if it has been executed within 30 seconds. If the participant was not able to stay inside the desired interval for at least two seconds, the task has been marked as timeout.

\begin{tabular}{|c|c|c|c|c|c|c|c|c|c|c|c|c|}
\hline & $\begin{array}{l}\text { (n) } \\
0 \\
. \\
. \\
0\end{array}$ & 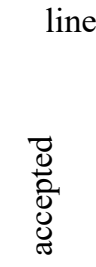 & 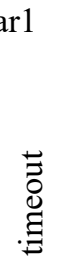 & 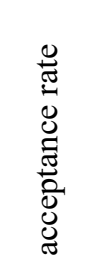 & $\begin{array}{l}\stackrel{\Xi}{\Xi} \\
\stackrel{Q}{\Xi}\end{array}$ & 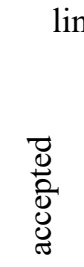 & 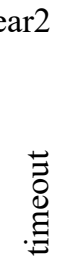 & 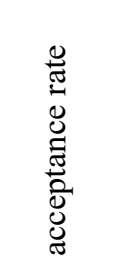 & $\stackrel{\stackrel{\Xi}{\Xi}}{\stackrel{\Xi}{Q}}$ & 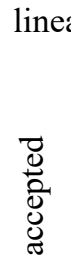 & 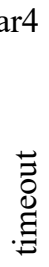 & 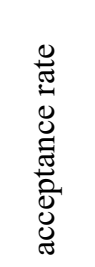 \\
\hline lateral axis (pitch) & $11,48 \mathrm{~s}$ & 121 & 9 & $93 \%$ & $5,97 \mathrm{~s}$ & 128 & 2 & $98 \%$ & $4,29 \mathrm{~s}$ & 130 & 0 & $100 \%$ \\
\hline longitudinal axis (roll) & $8,34 \mathrm{~s}$ & 126 & 4 & $97 \%$ & $5,09 \mathrm{~s}$ & 130 & 0 & $100 \%$ & $4,15 \mathrm{~s}$ & 130 & 0 & $100 \%$ \\
\hline perpendicular axis (yaw) & $6,10 \mathrm{~s}$ & 130 & 0 & $100 \%$ & $4,57 \mathrm{~s}$ & 130 & 0 & $100 \%$ & $3,74 \mathrm{~s}$ & 130 & 0 & $100 \%$ \\
\hline average & 8,64 & 125,7 & 4,3 & $96,7 \%$ & $5,21 \mathrm{~s}$ & 129,3 & 0,67 & $99,34 \%$ & $4,06 \mathrm{~s}$ & 130 & 0 & $100 \%$ \\
\hline
\end{tabular}

\begin{tabular}{|c|c|c|c|c|c|c|c|c|}
\hline & $\begin{array}{l}\stackrel{\mathscr{\Xi}}{\Xi} \\
\stackrel{Q}{Q}\end{array}$ & $\begin{array}{l}\vec{D} \\
\stackrel{0}{0} \\
\text { d } \\
0\end{array}$ & $\begin{array}{l}\vec{Z} \\
\stackrel{\Xi}{\Xi} \\
. \Xi\end{array}$ & 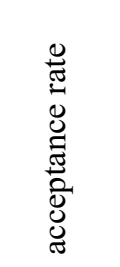 & $\stackrel{\stackrel{\Xi}{\Xi}}{\stackrel{\Xi}{Q}}$ & 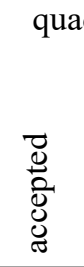 & 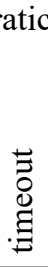 & 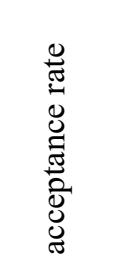 \\
\hline lateral axis (pitch) & $6,60 \mathrm{~s}$ & 128 & 2 & $98 \%$ & $7,64 \mathrm{~s}$ & 124 & 6 & $95 \%$ \\
\hline longitudinal axis (roll) & $5,93 \mathrm{~s}$ & 130 & 0 & $100 \%$ & $5,84 \mathrm{~s}$ & 130 & 0 & $100 \%$ \\
\hline perpendicular axis (yaw) & $4,90 \mathrm{~s}$ & 130 & 0 & $100 \%$ & $5,05 \mathrm{~s}$ & 130 & 0 & $100 \%$ \\
\hline average & $5,81 \mathrm{~s}$ & 129,3 & 0,67 & $99,34 \%$ & $6,17 \mathrm{~s}$ & 128,0 & 2 & $98,34 \%$ \\
\hline
\end{tabular}

linear 2 has been the fastest. Consequently, the smaller step size within the mobile environment leads to a higher granularity than the desktop waggle interaction.

Considering the absolute numbers in Tables 2 and 3, it can be ascertain, that the task completion on mobile devices has been twice as fast as the interaction using Leap Motion controller by comparing the best performance of each environments. In both studies, linear1 discretization has been the slowest, however in mobile environment, it reached similar times as the second best choice for desktop environments (linear2). Conclusive, it can be suggested, that the discretization size within the mobile device environment is not smaller than 2 and within the desktop environment not smaller than 4 . In none of the two environments, the discretization size based on sigmoid or quadratic functions as suggested by Rahman et al. [10], adduced an improvement in the time performance.

As we had not any timeouts in the study with mobile devices, the task acceptance rate is $100 \%$ for each discretization. On the contrary, using the Waggle on desktop, we could observe 23 timeouts overall, e.g.. for linear1, the acceptance rate is only $96,7 \%$. Therefore it can be assumed, that the interactions performed with the mobile device have a higher accuracy and higher efficiency that the performance on the desktop environment.

\section{Application Example: Factory planning}

Factory planning is characterized by the parallel consideration of multiple aspects such as production resources, production process and technology, and products, while anticipating uncertainty and future developments over the factory life-cycle [20]. These aspects usually result in different partial-models with specific information content (e.g., layout model, material flow model) and components of the factory (e.g. building, machinery, foundation, media), which need to be analyzed in combination. The different partial solutions are usually developed by various stakeholders, but typically interfere and require each other [18]. Appropriate visualization tools to support collaborative factory planning must be able to coordinate different layouts and viewpoints on the factory as well as exchange and 
manage information and models from different domains. VR-supported workflows are proposed to foster collaboration, establishment of a joint problem understanding, and exchange of different points of view [22].

Interesting views on the factory could be the following:

- Production facilities/areas: The arrangement of production areas can be analyzed if it is optimal (Figure 13 a).

- Material flow: Just as between the production areas, optimal material flow should be found for the working stations (Figure $13 \mathrm{~b}$ ).

- Workload of machines or workstations: Displaying the current utilization of the machines can signalize need for action or capabilities (Figure $13 \mathrm{c}$ ).

- Distances and dimensions: A user group in the factory layout planing are architects who particularly focus on the dimensions of machines and the distances between individual objects (Figure $13 \mathrm{~d})$.

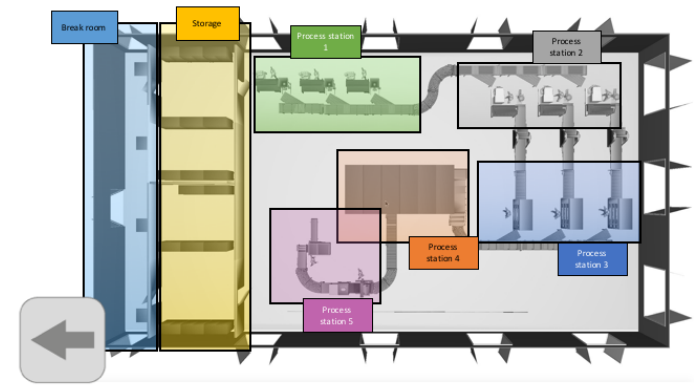

(a)

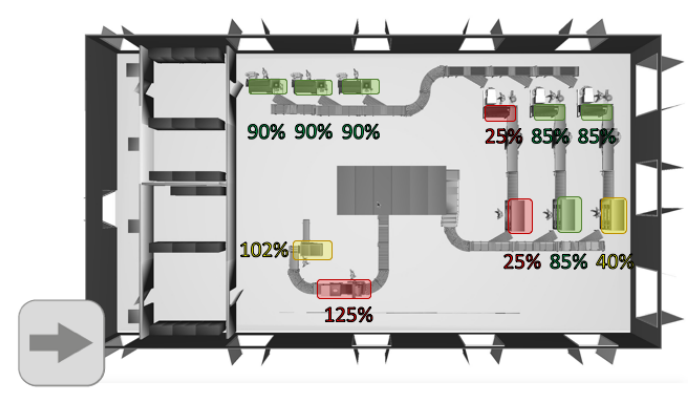

(c)

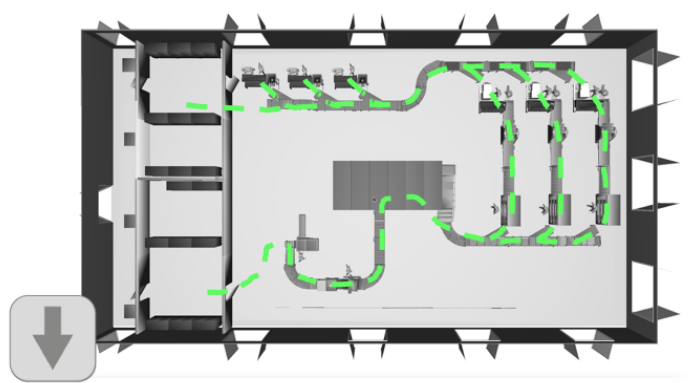

(b)

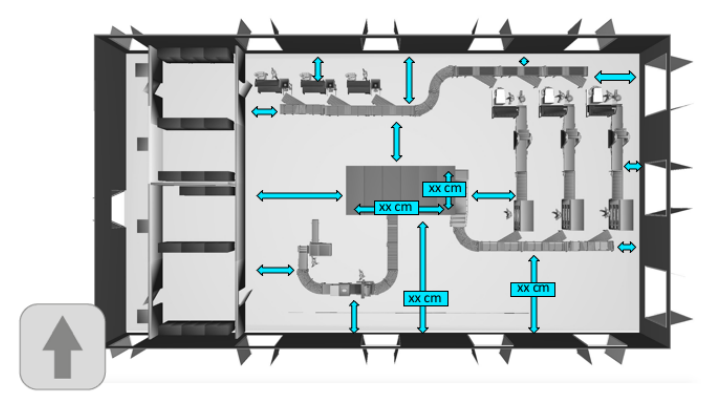

(d)

Fig. 13: In our factory planning use case, four different filters are implemented: (a) filter: production facilities, (b) filter: material flow, (c) filter: workload, (d) filter: distances. These filters can be accessed by tilting the device or hand left, right, up, and down.

These four views can be used to define the respective filters for the Waggle setup. Individually set, they describe the main task of the user group. However, dependencies between the different viewpoints exist. To represent these dependencies, filters can be combined (as in Fig. 14). For example, bottlenecks in the material flow in relation to the workload of the machines can easily be noticed and associated adjustments can be realized (see Fig. 14 c).

We implemented the waggle approach and these four views in a sample web-app that basically can be used in both environments, mobile and desktop (with slightly adapting the input mapping to the transparency degree). Test users were able to work with a prototype version of the application and 


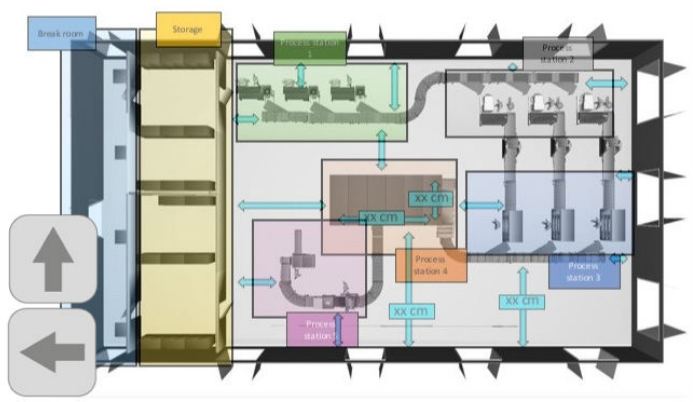

(a)

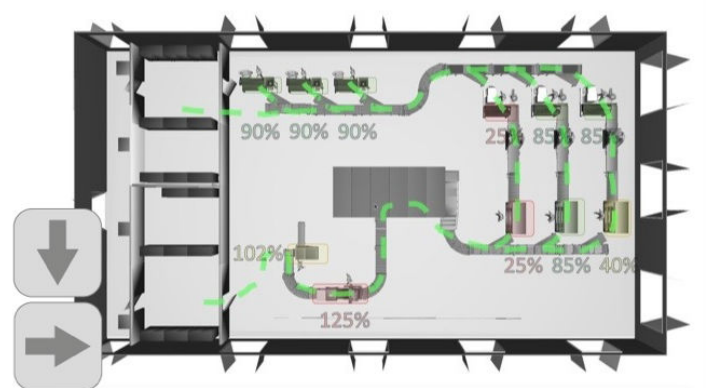

(c)

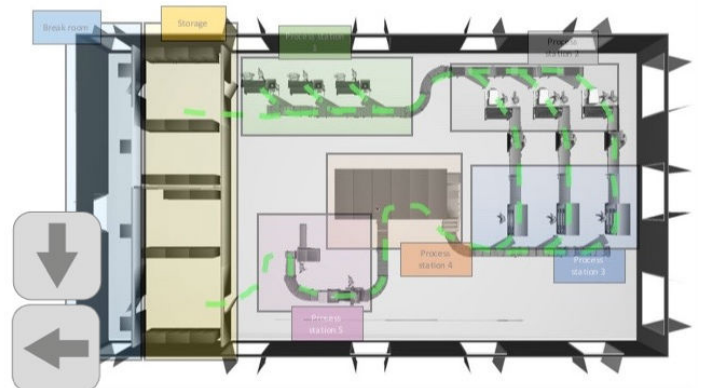

(b)

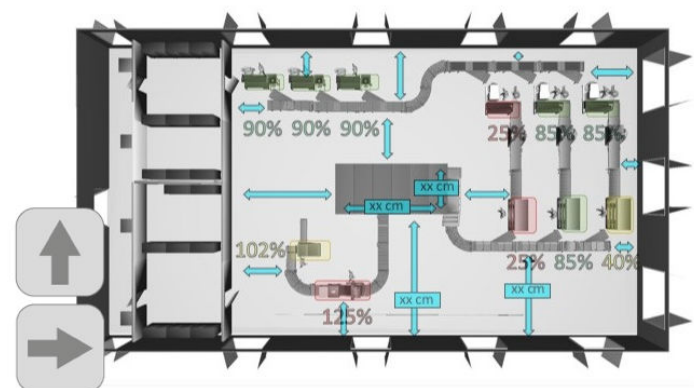

(d)

Fig. 14: Exemplary, combined views for factory layout planing tasks: (a) combined view from production facilities and distances, (b) combined view from production facilities and material flow, (c) combined view from material flow and workload, (d) combined view from workload and distances.

already gave encouraging feedback. Furthermore, they described the waggle metaphor as an intuitive and efficient approach. Therefore, we will continue to work on and enhance the application to conduct an extensive case study with it.

\section{Conclusion and Future Work}

In this paper we introduced the waggle metaphor, an interaction metaphor for enabling multiple views on underlying data across different devices. The orientation of device or hand measured by internal device sensors (accelerometer and gyroscope) or external sensors (Leap Motion controller) is used to provide touch-free interaction. In our two pilot studies, we investigated the range of motion and the discretization per waggle direction.

Table 4: Number of steps depending on range of motion and interval size.

\begin{tabular}{l|c|c|c|c|c|c} 
& \multicolumn{3}{|c|}{ Mobile } & \multicolumn{3}{c}{ Desktop } \\
& range of motion & interval size & steps & range of motion & interval size & steps \\
\hline lateral axis (pitch) & 32 & 2 & 16 & 41 & 4 & 10 \\
longitudinal axis (roll) & 40 & 2 & 20 & 60 & 4 & 15 \\
perpendicular axis (yaw) & 30 & 2 & 15 & 46 & 4 & 11 \\
\hline
\end{tabular}

In the first pilot study, we found that the range of motion is wider in desktop environment than in mobile environment. However, the second pilot study showed that a greater interval size is necessary to get faster selection in desktop environment. Dividing the possible range of motion by the interval size that we found in the second study, the number of steps in the range of motion is in fact less than 
in mobile environment for each of the waggle direction (see Table 4). As the total range of motion in desktop environment is larger, one might increase the interval as well to increase the efficiency. However, one side of each waggle direction ends at 9, 10, and 11 degrees respectively, hence, a larger interval of e.g. 8 would lead to only one step here. Overall, it would further decrease the number of steps.

It can be assumed, that the interactions performed with the mobile device have a higher accuracy and higher efficiency than the performance on the desktop environment. But this has to be tested in more detail and approved with the use of statistically formalisms.

In the desktop setting, the evaluation only studied the use of the right hand. In future work, we plan to conduct a study with the left hand and compare the results. This may have an effect on where to position the Leap Motion controller for effective and efficient integration in the daily work process. Furthermore, more complex tasks in different applications have to be implemented and evaluated, i.e. with respect to the assignments of different filters to different waggle directions and the user's ability to remember them.

To increase interaction possibilities in desktop environments, both hands could lead to higher granularity, e.g. adjusting numbers: use the left hand to configure the tens, and use the right hand to configure the units position. Furthermore, applying filters such as stabilization algorithms might increase the usability and the accuracy of the waggle metaphor in mobile and desktop environment as well. This has to be evaluated in the future work.

\section{Acknowledgements}

This research was partially funded by the German research foundation (DFG) within the IRTG 2057 "Physical Modeling for Virtual Manufacturing Systems and Processes" and the German Federal Ministry for Economic Affairs and Technology in the context of the technology program "Smart Data Innovations in Data", grant no. 01MD15004E.

\section{References}

[1] D. Bachmann, F. Weichert, and G. Rinkenauer. Evaluation of the leap motion controller as a new contact-free pointing device. Sensors (Basel, Switzerland), 15(1):214-233, 2015.

[2] M. Baglioni, E. Lecolinet, and Y. Guiard. Jerktilts: Using accelerometers for eight-choice selection on mobile devices. In Proceedings of the 13th International Conference on Multimodal Interfaces, ICMI '11, pages 121-128, New York, NY, USA, 2011. ACM.

[3] Ergo Vancouver. Wrist movements, 2017 (accessed February 06, 2017). http://www. ergovancouver.net/wrist_movements.htm.

[4] J. Guna, G. Jakus, M. Pogačnik, S. Tomažič, and J. Sodnik. An analysis of the precision and reliability of the leap motion sensor and its suitability for static and dynamic tracking. Sensors (Switzerland), 14(2):3702-3720, 2014.

[5] G. Jakus, J. Guna, S. Tomažič, and J. Sodnik. Evaluation of leap motion controller with a high precision optical tracking system. Lecture Notes in Computer Science (including subseries Lecture Notes in Artificial Intelligence and Lecture Notes in Bioinformatics), 8511 LNCS(PART 2):254-263, 2014.

[6] Leap Motion, Inc. Leapmotion, 2017 (accessed February 06, 2017). https://www.leapmotion. $\mathrm{com} /$. 
[7] G. Marin, F. Dominio, and P. Zanuttigh. Hand gesture recognition with leap motion and kinect devices. 2014 IEEE International Conference on Image Processing, ICIP 2014, pages 15651569, 2014.

[8] K. Partridge, S. Chatterjee, V. Sazawal, G. Borriello, and R. Want. Tilttype: Accelerometersupported text entry for very small devices. In Proceedings of the 15th Annual ACM Symposium on User Interface Software and Technology, UIST '02, pages 201-204, New York, NY, USA, 2002. ACM.

[9] L. E. Potter, J. Araullo, and L. Carter. The Leap Motion controller. Proceedings of the 25th Australian Computer-Human Interaction Conference on Augmentation, Application, Innovation, Collaboration - OzCHI '13, (February 2016):175-178, 2013.

[10] M. Rahman, S. Gustafson, P. Irani, and S. Subramanian. Tilt techniques: Investigating the dexterity of wrist-based input. In Proceedings of the SIGCHI Conference on Human Factors in Computing Systems, CHI '09, pages 1943-1952, New York, NY, USA, 2009. ACM.

[11] J. Rekimoto. Tilting operations for small screen interfaces. In Proceedings of the 9th Annual ACM Symposium on User Interface Software and Technology, UIST '96, pages 167-168, New York, NY, USA, 1996. ACM.

[12] A. Roudaut, M. Baglioni, and E. Lecolinet. TimeTilt: Using Sensor-Based Gestures to Travel through Multiple Applications on a Mobile Device, pages 830-834. Springer Berlin Heidelberg, Berlin, Heidelberg, 2009.

[13] J. Ryu, W. P. Cooney, L. J. Askew, K.-N. An, and E. Y. Chao. Functional ranges of motion of the wrist joint. The Journal of Hand Surgery, 16(3):409 - 419, 1991.

[14] J. Schwank, F.-A. Rupprecht, and A. Ebert. Waggle - orientation-based tablet interaction. In Proceedings of the 2017 CHI Conference Extended Abstracts on Human Factors in Computing Systems, CHI EA '17, New York, NY, USA, 2017. ACM. to be published.

[15] A. Scoditti, R. Blanch, and J. Coutaz. A novel taxonomy for gestural interaction techniques based on accelerometers. In Proceedings of the 16th International Conference on Intelligent User Interfaces, IUI '11, pages 63-72, New York, NY, USA, 2011. ACM.

[16] M. C. B. Seixas, J. C. S. Cardoso, and M. T. G. Dias. One Hand or Two Hands? 2D Selection Tasks With the Leap Motion Device. ACHI 2015 : The Eighth International Conference on Advances in Computer-Human Interactions, (c):33-38, 2015.

[17] M. C. B. Seixas, J. C. S. Cardoso, and M. T. G. Dias. The Leap Motion Movement for 2D Pointing Tasks - Characterisation and Comparison to Other Devices. Proceedings of the 5th International Conference on Pervasive and Embedded Computing and Communication Systems, pages 15-24, 2015.

[18] N. Shariatzadeh, G. Sivard, and D. Chen. Software evaluation criteria for rapid factory layout planning, design and simulation. Procedia CIRP, 3:299-304, 2012.

[19] J. Shen, Y. Luo, Z. Wu, Y. Tian, and Q. Deng. CUDA-based real-time hand gesture interaction and visualization for CT volume dataset using leap motion. Visual Computer, 32(3):359-370, 2016.

[20] T. Tolio, D. Ceglarek, H. ElMaraghy, A. Fischer, S. Hu, L. Laperrière, S. T. Newman, and J. Váncza. Species-co-evolution of products, processes and production systems. CIRP AnnalsManufacturing Technology, 59(2):672-693, 2010. 
[21] F. Weichert, D. Bachmann, B. Rudak, and D. Fisseler. Analysis of the accuracy and robustness of the Leap Motion Controller. Sensors (Switzerland), 13(5):6380-6393, 2013.

[22] C. Weidig, P. Galambos, Á. Csapó, P. Zentay, P. Baranyi, J. C. Aurich, B. Hamann, and O. Kreylos. Future internet-based collaboration in factory planning. Acta Polytechnica Hungarica, 11(7), 2014.

[23] Wikipedia. Lenticular Lens, 2016 (accessed March 29, 2016). https://en.wikipedia.org/wiki/ Lenticular_lens. 